\title{
The Ethics of Selecting for Disease Traits
}

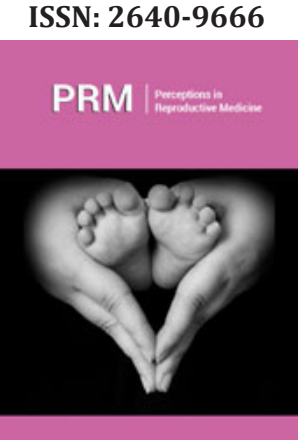

*Corresponding author: Ahmad Arabi, Department of Obstetrics and Gynecology, Wayne State University School of Medicine, Detroit, MI, USA

Submission: 侮 January 21, 2021

Published: 笽January 27, 2021

Volume 4 - Issue 2

How to cite this article: Ahmad Arabi, Brown K BS, Grimm L, Mowl S, Beltsos A, Jeelani R. The Ethics of Selecting for Disease Traits. Perceptions in Reproductive Medicine. 4(3). PRM. 000587. 2021.

DOI: 10.31031/PRM.2021.04.000587

Copyright@Ahmad Arabi, This article is distributed under the terms of the Creative Commons Attribution 4.0 International License, which permits unrestricted use and redistribution provided that the original author and source are credited.

\author{
Ahmad Arabi MD ${ }^{1 *}$, Brown K BS ${ }^{2}$, Grimm L MA ${ }^{3}$, Mowl S BS ${ }^{4}$, Beltsos A MD ${ }^{5}$ and \\ Jeelani R MD ${ }^{5}$ \\ ${ }^{1}$ Department of Obstetrics and Gynecology, Wayne State University School of Medicine, \\ Detroit, MI, USA \\ ${ }^{2}$ Wayne State University School of Medicine, Detroit, MI, USA \\ ${ }^{3}$ Rush University Medical College, Chicago, IL, USA \\ ${ }^{4}$ Department of Psychology, University of Michigan, Flint, MI, USA \\ ${ }^{5}$ Vios Fertility Institute, Chicago, IL, USA
}

\section{Abstract}

Background: While the discussion of allowing preimplantation genetic testing (PGT) with in vitro fertilization (IVF) to be utilized for selecting against disease traits is currently widely accepted, there is not yet a consensus regarding selection for an embryo with disease, or the high likelihood of developing disease. The use of PGT for disease selection is an ethical predicament that has not yet reached a unanimous consensus. The primary objective is to discuss arguments for and against the use of PGT for disease.

Main body: This article discusses arguments for and against the use of PGT for selecting for disease traits. Arguments against this practice include the principle of procreative beneficence and increased utilization of resources. Arguments for this practice include the principle of procreative autonomy, decreased risk of stigmatization, positive social impact, risk reduction methods, and the hope for future medical advancements.

Conclusion: There are multiple factors that must be considered before allowing parents to select for an embryo with disease, or high likelihood of developing disease, as this may result in serious implications for the child and society. It is imperative to keep in mind the balance between harm reduction and eugenics, as well as the potential impacts of prohibiting parents from choosing an embryo with a high probability of disease.

Keywords: Genetic selection;Ethics; PGT; IVF;Reproductive medicine;Genetics;Genetic disorders;Disease traits;Infertility.

\section{Background}

Preimplantation genetic testing (PGT) is often utilized in conjunction with in vitro fertilization (IVF) procedures to detect chromosomal abnormalities in embryos due to inherited structural rearrangements, such as translocations, inversions or duplications/ deletions, as well as age-related aneuploidy, thus improving live birth rates. It was first used in the early 1990s, to select for female embryos in couples at risk of transmitting recessive $\mathrm{X}$-linked diseases [1]. It has more recently been used to test for monogenic or single gene defects known to run in the family or for which the parents are known to be carriers [2]. This is an alternative to prenatal diagnosis and prevents unnecessary termination of pregnancy. It allows parents to avoid the long, anxious wait for results of post-implantation genetic testing while their fetus continues to grow. As the use of PGT increases, the weight of ethical concerns associated with it becomes more significant. The discussion of whether to allow PGT for selecting non-disease traits is well accepted and encouraged in society, but whether to allow parents to select for disease traits remained unanswered. It is a slippery slope between ensuring the best possible life for a child and eugenics, albeit the use of this term infers negative connotations and is controversial [3]. It is valuable to consider the implications of what occurs when a parent knowingly chooses an embryo that has a disease, or a high probability of having a disease, and what happens if parents are prohibited from, and possibly even penalized for, choosing to have a child that may potentially endure suffering.

\section{Main Text}

\section{Arguments against selecting disease traits}

Principle of procreative beneficence: Until CRISPR and similar genetic editing technologies become available, patients currently only choose from a menu of possible embryos. The idea of procreative beneficence states that from this menu of possible children, the one with the 
chance for the "best life" should be selected, regardless of whether or not disease related genes are being selected, and regardless of whether or not it leads to social inequalities [4]. According to this principle, if selection is possible, it is a moral obligation to select for the "most advantaged child" [5]. Parents have "significant moral reason" to use the available genetic information to select an embryo from their menu of possible embryos who is expected to have the best life [5]. The reasoning behind this principle is common sense morality. Selecting for better traits to provide benefit to the individual in terms of well-being is the moral thing to do. In this case, there is a conflict of principles between procreative autonomy and procreative beneficence. Autonomy would allow a couple with deafness to select for the deaf child if that is what they desire, while procreative beneficence would ensure the child without any hearing impairment is selected.

Resources needed for these diseases/financial burden/ services/therapies: When an individual has a chronic disease, extensive resources are needed to cover necessary medical treatment and therapies. If parents were to choose an embryo knowing that they will require extensive medical treatment and community resources, it would be an unethical burden on society. Resources could be better allocated to other conditions that are not preventable via PGT embryo selection. For example, those with cystic fibrosis, an incurable disease that can be screened for via IVF with PGT, have extensive medication and therapy needs and often require recurrent hospitalizations. In 2013, there were 12,590 hospital discharges with the main diagnosis of cystic fibrosis, with an associated cost of $\$ 94,664$ in average hospital charges per visit [6]. Given the challenges associated with cystic fibrosis, many patients and their families believe the disease is quite burdensome, despite progressing therapies that improve morbidity and mortality [7]. One study found that the majority of cystic fibrosis patients, as well as their parents, supported screening to avoid the birth of children with cystic fibrosis [7].

\section{Arguments for selecting for disease traits}

Procreative autonomy: Many bioethicists argue that the approach to reproductive ethical dilemmas should be guided by the concept of procreative autonomy. This principle states that parents should be free to make their own decisions about how, when, and where to reproduce, and what kind of children to have [8]. Within this principle, there is the exception that the state is to intervene only if there is significant evidence of harm to the patient, the child, or to society. The only reason for which an individual's liberty can be constrained is to protect other individuals. Although, there are further arguments that show how the risk to others can be mitigated.

Decreased stigmatization: Individuals who have developmental disabilities and/or chronic diseases experience significant stigmatization throughout their daily lives. Having a means to select against stigmatized traits further emphasizes the fact society does not want individuals with such traits to exist. When there is an underlying belief that an individual is unwanted, or a burden, the likelihood of abuse and neglect increases. As PGT becomes more readily available, it is expected to become a standard for family planning. It is of common belief that women should maintain proper health to improve the outcomes of their offspring, such as refraining from smoking and drinking alcohol, taking prenatal vitamins, eating a balanced diet, and attending prenatal doctor appointments. Such a belief may be extended upon women such that they are shamed for knowingly selecting an embryo with disease traits, or even pressured into PGT to guarantee their offspring have no abnormalities. Among a society where individuals with developmental abnormalities and chronic diseases already face stigmatization, the increased utilization of PGT for selection against disease traits could further ostracize those who have such conditions. Allowing for the selection of disease traits helps to reduce this stigmatization by showing these individuals that they are wanted and valued in our society.

Increased funding and support for those populations: If PGT were to become a common practice among all parents, the number of individuals with genetic diseases would significantly decrease. However, PGT can only confirm about $98 \%$ of disease traits, thus there will always be a small percentage of the population with disease [9]. When there is not a significant number of individuals with a disease, financial and academic resources are not dedicated to discovering a cure or advanced treatment for that disease. Not only would these individuals receive less medical support, they will likely experience an increased risk for psychological disorders. Mental health disorders, like anxiety, depression, and substance abuse, are significantly higher in those living with a rare chronic disease [10]. Therefore, the suffering of individuals with diseases will increase as selection against these diseases increases. Allowing parents to select for specific diseases will increase the overall population of diseased individuals with the benefit of increased funding and support for future therapeutic advancements.

Risk reduction methods: Although parents choosing to select disease traits are assuming the risk of raising a child who has a significant risk for suffering, there are preventative measures that can be taken to reduce these risks. For example, individuals with the BRCA1 or BRCA2 gene mutations, which increase the risk of breast and ovarian cancer, may take precautions to lower future risks by undergoing regular mammography, prophylactic mastectomy, and/ or prophylactic oophorectomy [11]. In addition, those with Down syndrome, who can experience mental and physical disabilities, may participate in early intervention programs aimed at improving fine motor skills, [12] strength, balance, [13] and speech and language skills [14]. Risk reduction methods such as these may lessen or negate the concerns of future children experiencing significant suffering.

Future medical advancements: Along with risk reduction methods, there is no certainty of the medical advancements that could be established throughout the lifetime of the affected child. 
There will likely be new medical treatments that would improve the quality of life of those affected children. Life expectancies continue to rise as new medical knowledge increases at an exponential rate. For instance, the mortality of cystic fibrosis decreased by $49.3 \%$ between 2003 and 2013 [6]. Moreover, treatment options for Huntington disease are rapidly evolving, with multiple promising therapeutic options under investigation [15]. While future medical advances are not a certainty, there is continuously new research being performed in hopes of discovering novel medical treatments that improve the lives of those with chronic disease. These medical advances have the potential to greatly lessen, or completely remove, any negative impact that a disease trait has on an individual.

\section{Discussion}

There are clearly compelling arguments both for and against the practice of selecting for disease traits in an embryo. The ethical principle of procreative autonomy, decreasing risk of stigmatization, increasing funding and support, as well as the possibility of utilizing risk reduction methods and future medical advances, may be used to justify the selection of embryos with disease traits. However, parents who purposefully choose an embryo with a high risk of disease potentially violate the principle of procreative beneficence while also increasing their future child's burden on society. As the utilization of PGT increases as is expected, this moral issue becomes even more pressing. It is a widely held sentiment that most parents do not wish to cause their children unnecessary harm. The discussion arises, however, when we aim to determine what qualifies as harm and what does not. For example, while some may see deafness as a negative trait, others value deafness and the culture associated with it and would prefer to have a deaf child [16]. Individuals with inherited diseases are still able to live fulfilling lives, and it may be ableist to say that these diseases take away from their quality of life. It is a slippery slope from harm reduction to eugenics and, essentially, choosing who is and is not allowed to live and reproduce. Eugenics is defined as an attempt to improve the human gene pool [17]. However, many find the term morally objectionable because they associate it with authoritarian coercive methods and unjustifiable practices [3]. Care must be taken when using the term eugenics as it provides a stern connotation before we even start to consider the issues. This may lead to unnecessary and unjustifiable harm to patients who stand to benefit from these new interventions [3].

One compromise between the arguments for and against selecting for a disease trait would be that choosing embryos with certain, milder traits is ethical, while selecting for more severe, debilitating traits is not. However, we are then faced with the challenge of determining which traits fall under each category, who is charged with drawing this line, and how this will be enforced. If it is deemed unethical to select for an embryo with a disease, or high likelihood of contracting a disease, parents may be restricted from doing so. While in the United States there are currently no legal requirements regarding prenatal testing, there are nonbinding clinical practice guidelines that support widespread use of certain testing, as put forth by professional and scientific societies such as American College of Obstetrics and Gynecology [18]. These guidelines aim to direct clinical practice and are often referenced by health care professionals, as well as cited in malpractice cases. If selecting for disease traits utilizing PGT was recommended against by these societies, health care providers may then refuse to provide this service to their patients. While this would not be a legal prohibition, this would still entail the reality that parents would be unable to select for an embryo with a disease trait. In addition to violating the parents' procreative autonomy, this may push individuals towards foregoing PGT in hopes of selecting an embryo with a certain disease trait by chance. It has been shown that IVF with PGT for aneuploidy reduces healthcare costs, treatment time, risk of failed embryo transfer, and risk of clinical miscarriage [19]. Foregoing this testing would thereby increase these costs and risks. This raises its own moral question; if selecting for disease traits is deemed unethical, is refusing to test for these traits then also unethical?

Currently, some parents of children with genetic conditions are not interested in genetic testing for subsequent pregnancies. These choices may be impacted by lack of confidence in the accuracy of medical tests, situational factors regarding their particular clinical environment, age, ethnicity, income, religious beliefs, and philosophical beliefs [20]. Parents may also wish to forego prenatal testing as the results, whether positive or negative, would not change their desire to continue the pregnancy to term [21]. Forcing parents to undergo prenatal genetic testing that they would rather refuse would violate the ethical principle of procreative autonomy, just as regulating which embryos they may select for using PGT would.

\section{Summary}

While the discussion of allowing PGT with IVF to be utilized for selecting against disease traits is currently widely accepted, there is not yet a consensus regarding selecting for an embryo with disease, or the high likelihood of developing disease. Arguments against this practice include the principle of procreative beneficence and increased utilization of resources. Arguments for this practice include the principle of procreative autonomy, decreased risk of stigmatization, positive social impact, risk reduction methods, and the hope for future medical advances. It is also imperative to keep in mind the balance between harm reduction and eugenics, as well as the potential impacts of prohibiting parents from choosing an embryo with a high probability of disease.

\section{References}

1. Handyside AH, Kontogianni EH, Hardy K, Winston RM (1990) Pregnancies from biopsied human preimplantation embryos sexed by Y-specific DNA amplification. Nature 344(6268): 768-770.

2. Daar J, Benward J, Collins L, Davis J, Davis O, et al. (2018) Use of preimplantation genetic testing for monogenic defects (PGT-M) for adult-onset conditions: An Ethics Committee opinion. Fertil Steril 109(6): 989-992. 
3. Wilkinson S, Garrard E (2001) Eugenics and the ethics of selective reproduction. Keele University, UK.

4. Savulescu J (2001) Procreative beneficence: Why we should select the best children. Bioethics 15(5-6): 413-426.

5. Savulescu J, Kahane G (2009) The moral obligation to create children with the best chance of the best life. Bioethics 23(5): 274-290.

6. Agarwal A, Agarwal A, Mehta D, Sikachi RR, Du D, et al. (2017) Nationwide trends of hospitalizations for cystic fibrosis in the United States from 2003 to 2013. Intractable \& Rare Diseases Research 6(3): 191-198.

7. Janssens S, Chokoshvilli D, Binst C, Mahieu I, Henneman L, et al. (2016) Attitudes of cystic fibrosis patients and parents toward carrier screening and related reproductive issues. Eur J Hum Genet 24(4): 506-512.

8. Caplan A (1999) The future of human reproduction: Ethics, choice, and regulation. BMJ 318(7188): 948A.

9. (2020) Preimplantation genetic testing: ACOG Committee Opinion, Number 799. Obstet Gynecol 135(3): e133-e137.

10. Nunn R (2017) It's not all in my head!-The complex relationship between rare diseases and mental health problems. Orphanet J Rare Dis 12(1): $1-3$.

11. Kurian AW, Sigal BM, Plevritis SK (2010) Survival analysis of cancer risk reduction strategies for BRCA1/2 mutation carriers. J Clin Oncol 28(2): 222-231.

12. Aparicio TS, Balaña JM (2009) A study of early fine motor intervention in down's syndrome children. Early Child Development and Care 179(5): 631-666.
13. González LR, Antón LD, Salazar A, Valero MR, Munoz MJ (2019) Physical therapy in down syndrome: Systematic review and meta-analysis. J Intellect Disabil Res 63(8): 1041-1067.

14. Prèvost L (2002) Speech and language therapy for children with down syndrome. Down Syndrome News and Update 2(2): 70-76.

15. Potkin KT, Potkin SG (2018) New directions in therapeutics for huntington disease. Future Neurol 13(2): 101-121.

16. Wallis JM (2020) Is it ever morally permissible to select for deafness in one's child? Med Health Care Philos 23(1): 3-15.

17. Chadwick R (1998) Genetics and ethics. Routledge encyclopedia of philosophy London: Routledge, UK.

18. Pergament D, Ilijic K (2014) The legal past, present and future of prenatal genetic testing: Professional liability and other legal challenges affecting patient access to services. J Clin Med 3(4): 1437-1465.

19. Neal SA, Morin SJ, Franasiak JM, Goodman LR, Juneau CR, et al. (2018) Preimplantation genetic testing for aneuploidy is cost-effective, shortens treatment time, and reduces the risk of failed embryo transfer and clinical miscarriage. Fertil Steril 110(5): 896-904.

20. Kelly SE (2009) Choosing not to choose: Reproductive responses of parents of children with genetic conditions or impairments. Sociol Health Illn 31(1): 81-97.

21. Decruyenaere M, Kiebooms EG, Boogaerts A, Philippe K, Demyttenaere $\mathrm{K}$, et al. (2007) The complexity of reproductive decision-making in asymptomatic carriers of the Huntington mutation. Eur J Hum Genet 15(4): 453-462. 